\section{RMD Open}

Rheumatic \&

Musculoskeletal Diseases

To cite: Hedström AK, Åkerstedt T, Klareskog L, et al. Relationship between shift work and the onset of rheumatoid arthritis. RMD Open 2017;3:e000475. doi:10.1136/ rmdopen-2017-000475

- Prepublication history for this paper is available online. To view these files please visit the journal online (http://dx.doi. org/10.1136/rmdopen-2017000475).

Received 23 April 2017

Revised 28 June 2017

Accepted 4 July 2017

\section{CrossMark}

${ }^{1}$ Institute of Environmental Medicine, Karolinska Institutet, Stockholm, Sweden

${ }^{2}$ Stress Research, Stockholm University, Stockholm, Sweden ${ }^{3}$ Department of Clinical Neuroscience, Karolinska Institutet, Stockholm, Sweden

${ }^{4}$ Rheumatology Unit, Department of Medicine, Karolinska University Hospital, Stockholm, Sweden

Correspondence to Dr Anna Karin Hedström; anna.hedstrom@ki.se

\title{
Relationship between shift work and the onset of rheumatoid arthritis
}

\author{
Anna Karin Hedström, ${ }^{1}$ Torbjörn Åkerstedt, ${ }^{2,3}$ Lars Klareskog, ${ }^{4}$ Lars Alfredsson ${ }^{1}$
}

\section{ABSTRACT}

Background Environmental factors play a prominent role in rheumatoid arthritis (RA) aetiology. Shift work has previously been associated with increased RA risk in females. The aim of this study was to investigate the potential association, including a dose-response association, between permanent night shift work, rotating shift work and day-oriented shift work and risk of developing anticitrullinated peptide antibodies (ACPA)positive and ACPA-negative RA.

Methods The present report is based on a populationbased, case-control study with incident cases of RA (1951 cases and 2225 controls matched by age, gender and residential area). Using logistic regression, occurrence of RA among subjects who have been exposed to different kinds of shift work was compared with that among those who have never been exposed by calculating the OR with a $95 \% \mathrm{Cl}$.

Results Rotating shift work and day-oriented shift work increased the risk of developing ACPA-positive RA (OR 1.3, $95 \% \mathrm{Cl} 1.0$ to 1.7 and $\mathrm{OR} 1.3,95 \% \mathrm{Cl} 1.0$ to 1.6 ), but not ACPA-negative RA. Permanent night shift work appeared to be a protective factor both against ACPA-positive RA (OR $0.7,95 \% \mathrm{Cl} 0.6$ to 0.9 ) and ACPA-negative RA (OR $0.8,95 \% \mathrm{Cl} 0.6$ to 1.0 ). For both subsets of RA, significant trends showed a lower risk of developing RA with increasing duration of permanent night shift work ( $p$ value for trend 0.002 vs 0.04 ).

Conclusions Sleep restriction as a consequence of shift work is associated with several biological effects among which changes in melatonin production may be involved. The present epidemiological findings of a complex relationship between sleep patterns and different forms of RA may be of importance for increasing the understanding of the pathophysiology of RA.

\section{INTRODUCTION}

Rheumatoid arthritis (RA) is a chronic inflammatory joint disease that shows rhythmic variations in clinical symptoms depending on the biological clock. ${ }^{1}$ Susceptibility to RA is determined by both genetic and environmental factors. The disease is subclassified into two subsets based on the presence of anticitrullinated peptide antibodies (ACPA) ACPAs are specific markers of RA that occur in approximately $60 \%$ of the patients. ${ }^{23}$ Several reported susceptibility genes for RA including the human leukocyte antigen (HLA) DRB1

\section{Key messages}

What is already known about this subject?

- All types of shift work have been associated with long-term sleep restriction, which may lead to persistent changes in the immune system and enhanced proinflammatory responses.

- Shift work, defined as working outside regular day shifts, has previously been associated with increased risk of developing rheumatoid arthritis (RA) in females.

- Melatonin production is decreased in permanent night shift workers, and several lines of evidence point to a disease-promoting role of melatonin in $\mathrm{RA}$, and it is therefore of interest to investigate the impact of different kinds of shift work on RA risk.

What does this study adds?

- Rotating shift work and day-oriented shift work was associated with increased risk of anticitrullinated peptide antibodies (ACPA)-positive RA, but not ACPAnegative $R A$.

- Permanent night shift work was inversely associated with both subsets of RA and significant trends showed a lower risk of developing $R A$ with increasing duration of permanent night shift work

How might this impact on clinical practice?

- The present epidemiological findings of a complex relationship between sleep patterns and different forms of RA may be of importance for increasing the understanding of the pathophysiology of RA and providing a basis for information to patients who have questions about this topic.

shared epitope (SE) alleles have been associated with ACPA-positive RA, but not with ACPA-negative RA. ${ }^{4}$ Similarly, several environmental risk factors have a more pronounced influence on ACPA-positive RA, such as smoking ${ }^{67}$ and exposure to silica. ${ }^{8}$

Mounting evidence indicates that shift work is associated with a wide variety of adverse health consequences. Shift work has been linked with increased risk of developing autoimmune thyroid disorders, ${ }^{9}$ multiple sclerosis, ${ }^{10} 11$ diabetes, ${ }^{12}$ cardiovascular disease ${ }^{13}$ and cancer. ${ }^{14}$ In a prospective cohort study, shift work defined as working outside regular 
day shifts, was associated with an increased risk of developing RA in females (OR $1.3,95 \%$ CI 1.0 to 1.8 ). ${ }^{15}$ However, immune processes and clinical symptoms of RA follow a circadian rhythm, and there is a bidirectional communication between the circadian and the immune system involved in RA pathogenesis. ${ }^{16} 17$ Permanent night shift work, but not other kinds of shift work, decreases the production of melatonin, a hormone produced by the pineal gland which may have a disease-promoting role in RA pathogenesis. ${ }^{18-22}$ We therefore hypothesise that different kinds of shift work may influence the risk of RA in different ways. Since several environmental risk factors have a more pronounced influence on ACPA-positive RA, we aimed to explore the influence of permanent night shift work, rotating shift work and day-oriented shift work on the risk of developing ACPA-positive and ACPA-negative RA.

\section{METHOD}

\section{Design and study population}

This report was based on data from the ongoing project Epidemiological Investigation of Rheumatoid Arthritis (EIRA) which is a population-based case-control study comprising the population aged 18-70 years in the middle and southern parts of Sweden. All hospital-based and most privately run rheumatology units participated in recruiting cases of RA to the study. For each potential case, one control was randomly selected from the national population register, matched by age, gender and residential area. Cases who did not fulfil the American College of Rheumatology 1987 criteria at the time of the analyses were excluded, whereas we kept the corresponding controls.

During the study period June 1996 to December 2006, completed questionnaires were obtained from 1990 cases who fulfilled the American College of Rheumatology 1987 criteria and 2230 controls; the response proportion being $92 \%$ for the cases and $75 \%$ for the controls. ACPA status was missing in 34 cases, and these were excluded. Five cases and five controls who reported shift work were excluded since they could not specify during which years they were exposed. The present study thus comprised 1951 cases and 2225 controls. All aspects of the study were approved by the ethics committee of the Karolinska Institutet.

\section{Anticitrullinated peptide antibodies}

ACPA status among cases was analysed using Immunoscan RA Mark 2 ELISA test (anti-CCP2 test). ${ }^{23}$. Serum samples were diluted $1 / 50$ with dilution buffer and incubated for 1 hour at $37^{\circ} \mathrm{C}$. After removing the liquid and washing three times with rinsing buffer, the conjugate solution (peroxidase conjugated anti-human IgG antibodies) was added into each well and incubated for 1 hour at $37^{\circ} \mathrm{C}$. After three washing steps with rinsing buffer, the substrate solution (tetramethyl benzidine) was added and incubated for $30 \mathrm{~min}$ at room temperature. The stop solution (sulfuric acid, $0.5 \mathrm{~mol} / \mathrm{L}$ ) was added and the absorbance values were read immediately at $450 \mathrm{~nm}$. An antibody level exceeding $25 \mathrm{AU} / \mathrm{ml}$ was regarded as ACPA positivity.

\section{Data collection and definition of shift work}

The questionnaire contained questions relating to demographic and reproductive factors, heredity, previous health, lifestyle factors, occupational exposures and socioeconomic circumstances. Detailed information was obtained regarding professions and work places, including whether subjects had worked outside standard working hours. Subjects could in detail specify type of shift work by answering a free text question and specify during which periods they had worked shift. Incompletely answered questionnaires were completed by mail or by telephone. For each case, the time of the initial appearance of RA symptoms was used as an estimate of the disease onset, and the year in which this occurred was defined as the index year. The corresponding control was given the same index year. Information regarding shift work was considered prior to the index year in the cases and during the same period of time in the corresponding controls.

Shift work comprises work schedules that extend beyond the typical nine-to-five workday. In the present study, shift workers were categorised based on their schedules into those who reported day-oriented shift work, rotating shift work and permanent night shift work. Subjects with morning and evening work, in which short daily rest between shifts is common, were included in the first group. Rotating shift work included those who had worked both day time and night time working hours, those who had worked rotating day and night shifts and those who worked 24 hours shifts. Permanent night shift work comprised those who exclusively had worked night shifts. In the main analysis, subjects who had been exposed to different kinds of shift work were categorised based on what kind of shift work took place closest to the index year. We also performed complementary analyses defining permanent night shift work as ever having worked permanent night shifts before the index year and defining shift work as ever having worked either day oriented or rotating shift before the index year. In order to analyse the influence of duration and intensity of the different kinds of shift work, we further categorised the subjects into subgroups based on the duration $(<5$, $5-10,>10$ years) and intensity ( $\leq 10$ or $>10$ nights $/$ month) of the shift work.

\section{Statistical analysis}

By means of logistic regression, the incidence of ACPA-positive and of ACPA-negative RA, respectively, in subjects who had worked permanent night shift work, day-oriented shift work or rotating shift work at the index year were compared with those who had never worked shifts, by calculating ORs with $95 \%$ CIs. The analysis was also performed separately for men and women. Trend 
test for a dose-response relationship regarding duration and intensity of permanent night shift work and risk of RA was performed by using a continuous variable for duration and intensity of permanent night shift work, in a logistic regression model.

Both matched and unmatched analyses of the data were performed. However, only the results from the unmatched analyses are presented in this report since these were in close accordance with those from the matched analyses but in general had higher precision in terms of more narrow CIs.

All analyses were adjusted for age, gender, residential area, ancestry and smoking habits. Age was adjusted for as a continuous variable. Assessment of ancestry was based on whether the subject was born in Sweden or not and whether either of the subject's parents had immigrated to Sweden. A subject who was born in Sweden, whose parents had not immigrated, was classified as Swedish. Smoking was adjusted for as a continuous variable based on number of pack years. One pack year is equivalent to smoking 20 cigarettes daily for 1 year. Adjustments were also made for educational level (university degree or not), body mass index at inclusion in the study (underweight, normal weight, overweight or obese) and alcohol consumption (gram alcohol per week at study inclusion), but these factors did not influence on the results and were not retained in the final analyses. The complementary analyses were adjusted for shift work versus permanent shift work. All analyses were conducted using Statistical Analysis System (SAS) V.9.2.

\section{RESULTS}

The analyses of shift work and RA risk included 1951 cases and 2225 controls. The mean age at onset was 50.5 years for ACPA-positive cases and 51.8 years for ACPA-negative subjects. The mean duration from the disease onset to inclusion in the study was less than 1 year in both subgroups (0.9 years, SD 1.3 for ACPA-positive RA and 0.9 years, SD 1.5 for ACPA-negative RA). Characteristics of cases and controls are presented in table 1 .

There was a significant association between both rotating shift work and day-oriented shift work and increased incidence of ACPA-positive RA, but not ACPA-negative RA. The adjusted OR of developing ACPA-positive RA was 1.3 (95\% CI 1.0 to 1.6) among those who had worked rotating shifts and $1.3(95 \%$ CI 1.0 to 1.7$)$ among those who had worked day-oriented shifts, compared with those who had never been exposed to any kind of shift work (table 2). There were no gender differences regarding association between different kinds of shift work and RA risk. The results were similar when we defined shift workers as ever having worked day oriented or rotating shifts and adjusted the analysis for permanent night shift work (adjusted OR of ACPA-positive RA was 1.3 (95\% CI 1.1 to 1.6$)$ and adjusted OR of ACPA-negative RA was 1.0 (95\% CI 0.8 to 1.3) for those who had been exposed to day oriented

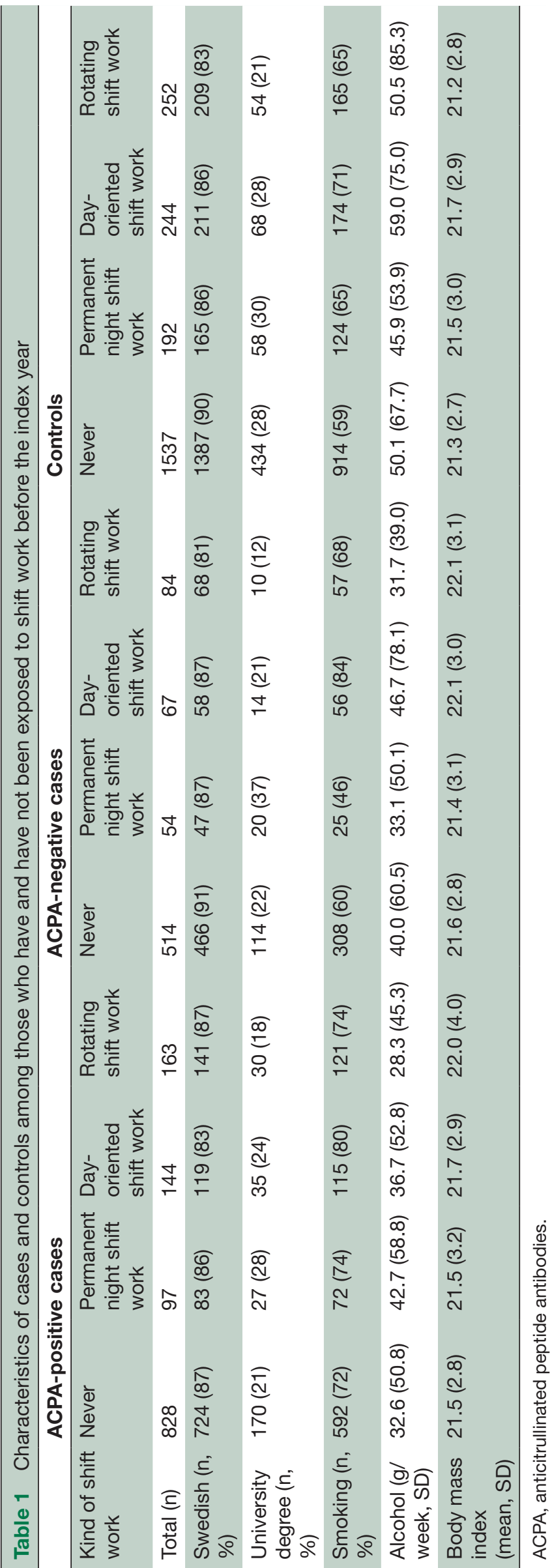


Table 2 ORs with 95\% Cls of developing rheumatoid arthritis (RA) for subjects who have been exposed to permanent night shift work, rotating shift work, or day oriented shift work, compared with those who have never been exposed

\begin{tabular}{|c|c|c|c|c|c|c|}
\hline & & ACPA-positive & RA & & $\begin{array}{l}\text { ACPA- } \\
\text { negative RA }\end{array}$ & \\
\hline Type of shift work & $\mathrm{ca} / \mathrm{co}^{*}(\mathrm{n}, \%)$ & OR $(95 \% \mathrm{Cl}) \dagger$ & $p$ Value & $\mathrm{ca} / \mathrm{co}^{\star}(\mathrm{n}, \%)$ & OR $(95 \% \mathrm{Cl}) \dagger$ & $p$ Value \\
\hline Never & $828(67) / 1537(69)$ & 1.0 (reference) & & $514(71) / 1537(69)$ & 1.0 (reference) & \\
\hline Day oriented shift work & $144(12) / 244(11)$ & $1.3(1.0$ to 1.7$)$ & 0.04 & $67(9) / 244(11)$ & $1.0(0.7$ to 1.4$)$ & 0.9 \\
\hline Rotating shift work & $163(13) / 252(11)$ & 1.3 (1.0 to 1.6$)$ & 0.03 & $84(12) / 252(11)$ & 1.1 (0.9 to 1.5$)$ & 0.4 \\
\hline Permanent night shift work & $97(8) / 192(9)$ & 0.7 (0.6 to 0.9$)$ & 0.005 & $54(8) / 192(9)$ & 0.8 (0.6 to 1.0$)$ & 0.03 \\
\hline
\end{tabular}

*Exposed cases and controls

†Adjusted for age, gender, residential area, ancestry, and smoking.

ACPA, anticitrullinated peptide antibodies.

or rotating shifts compared with those who have never been exposed).

There was an inverse association between permanent night shift work and risk of each subset of RA. Compared with those who had never been exposed to shift work, the adjusted OR of developing ACPA-positive RA was 0.7 (95\% CI 0.6 to -0.9 ) and the adjusted OR of developing ACPA-negative RA was 0.8 (95\% CI 0.6 to 1.0) for those who had worked permanent night shifts before the index year (table 2). There were no gender differences. The results were similar when we defined permanent night shift work as ever having worked permanent night shifts before the index year and adjusted the analysis for day oriented or rotating shift work (adjusted OR of ACPA-positive RA was 0.7 (95\% CI 0.6 to 0.9 ) and adjusted OR of ACPA-negative RA was 0.8 (95\% CI 0.6 to 1.0 ) for those who had been exposed to permanent night shift work compared with those who had never been exposed).

For both subsets of RA, significant trends showed a lower risk of developing RA with increasing duration of permanent night shift work (table 3). For ACPA-positive
RA, there was also a significant inverse trend between intensity of permanent night shift work and RA risk.

\section{DISCUSSION}

In accordance with a previous prospective study on shift work and RA risk, ${ }^{15}$ we observed an association between shift work and increased RA risk. Compared with subjects who had never worked shifts, the risk of ACPA-positive RA was increased by $30 \%$ both among those who had worked day-oriented shift work and among those who had worked shifts including both daytime and night time working hours. There was no association between risk of ACPA-negative RA and shift work.

Sleep restriction may be a possible mechanism behind the association between shift work and RA risk. All types of shift work may result in sleep restriction, ${ }^{24}$ and mounting evidence indicates that shift work is associated with a wide variety of adverse health consequences. Sleep deprivation has itself been shown to severely disturb the functional rhythm of regulatory $\mathrm{T}$ cells and $\mathrm{CD} 4+\mathrm{T}$

Table 3 ORs with $95 \% \mathrm{Cl}$ of developing rheumatoid arthritis (RA) for subjects who reported past exposure to permanent night shift work compared with those who had never been exposed, by duration (years) and intensity (nights/months) of night shift work

\begin{tabular}{|c|c|c|c|c|c|c|}
\hline \multicolumn{4}{|l|}{ ACPA-positive RA } & \multicolumn{3}{|c|}{ ACPA-negative RA } \\
\hline Duration & $\mathrm{ca} / \mathrm{co}^{*}$ & OR $(95 \% \mathrm{Cl}) \dagger$ & $p$ Value & $\mathrm{ca} / \mathrm{co}^{*}$ & OR $(95 \% \mathrm{Cl}) \dagger$ & $p$ Value \\
\hline 0 & $828 / 1537$ & 1.0 (reference) & & $514 / 1537$ & 1.0 (reference) & \\
\hline$<5$ years & $149 / 275$ & 0.8 (0.6 to 1.0$)$ & 0.06 & $81 / 275$ & 0.9 (0.7 to 1.2$)$ & 0.5 \\
\hline $5-10$ years & $45 / 89$ & 0.7 (5.1 to 1.1$)$ & 0.1 & $24 / 89$ & 0.8 (0.5 to 1.3$)$ & 0.4 \\
\hline$>10$ years & $65 / 152$ & 0.6 (0.4 to 0.9$)$ & 0.005 & $35 / 152$ & 0.7 (0.4 to 1.0$)$ & 0.06 \\
\hline$p$ for trend & & & 0.002 & & & 0.04 \\
\hline Intensity & $\mathrm{ca} / \mathrm{co}^{*}$ & OR $(95 \% \mathrm{Cl}) \dagger$ & $\mathrm{p}$ & $\mathrm{ca} / \mathrm{co}^{*}$ & OR $(95 \% \mathrm{Cl}) \dagger$ & $p$ \\
\hline 0 & $828 / 1537$ & 1.0 (reference) & & $514 / 1537$ & 1.0 (reference) & \\
\hline$\leq 10$ nights/months & $184 / 362$ & $0.8(0.6$ to 1.0$)$ & 0.02 & $97 / 362$ & 0.8 (0.6 to 1.1$)$ & 0.1 \\
\hline$>10$ nights/months & $75 / 154$ & 0.7 (0.5 to 1.0$)$ & 0.03 & $43 / 154$ & 0.8 (0.6 to 1.2$)$ & 0.4 \\
\hline $\mathrm{p}$ for trend & & & 0.007 & & & 0.2 \\
\hline
\end{tabular}

${ }^{*}$ Number of exposed cases and controls.

†Adjusted for age, gender, residential area, ancestry, smoking, educational level and shift work.

ACPA, anticitrullinated peptide antibodies. 
cells. ${ }^{25}$ It has been hypothesised that clock desynchrony in the leucocyte subpopulations due to chronic sleep disturbances will promote immune-related diseases such as autoimmunity, allergy and tumours. ${ }^{26}$ Sleep deprivation is also associated with elevation of proinflammatory cytokines such as interleukin (IL)-6 and tumour necrosis factor-alpha, which have been proposed as mediators of the negative consequences of shift work. ${ }^{27}$ Further, longterm sleep restriction may lead to persistent changes in the immune system and an increased production of IL-17. ${ }^{28}$ Th17 cells have been shown to play a crucial role in the induction of autoimmune diseases such as RA. ${ }^{29}$

Permanent night shift work was inversely associated with both subsets of RA. Among night shifts workers, the OR of developing either of the two subsets of RA was reduced by $30 \%$ compared with those who had never worked night shifts. Sleep restriction is a consequence also of permanent night shift work. However, a possible contributing factor behind the inverse relationship between permanent night shift work and RA risk may be reduced melatonin production in night shift workers. Permanent night shift workers have lower levels and a delay in peak time of melatonin production over a 24-hours period. ${ }^{18-21}$ Melatonin production decreased progressively over consecutive days of simulated night work, both during night time and over the 24 hours. ${ }^{22}$ Several lines of evidence point to a disease-promoting role of melatonin in RA. Macrophages infiltrating the synovial fluid of patients with RA have specific melatonin-binding sites, and melatonin has also been found at high concentrations in the synovial fluid..$^{30}$ Compared with healthy controls, patients with RA have altered levels of circulating melatonin with higher nocturnal serum levels of melatonin and an altered temporal profile with a more rapid increase at the start of the night and an earlier peak. ${ }^{31}$ The circadian serum concentrations of melatonin were also significantly higher in northern than in southern patients and the geographical distribution of RA shows a northsouth gradient, with higher latitudes being associated with an increased incidence and severity of RA. ${ }^{32}$ Furthermore, a large number of studies in animal models of RA indicate that melatonin promotes the development or increases the severity of RA. ${ }^{33}{ }^{34}$ The decreased melatonin production associated with night shift work may thus contribute to explaining the inverse relationship between night shift work and risk of developing RA.

The observation that permanent night work seems to protect against RA needs corroboration from subsequent epidemiological studies. However, there is also a need for field studies and experiments with night work, with a particular focus on the measurement of levels of melatonin across shift cycles. The experiments should also include patients with RA whose sleep is shifted to daytime, while both melatonin levels and symptoms are recorded. Finally, ways of blocking melatonin, for example, with light should be attempted. This might include optimising split sleep strategies with light exposure during melatonin peak time.
EIRA was designed as a population-based, case-control study using incident cases. Information regarding lifestyle factors and personal information was gathered retrospectively. However, we predominantly included cases of RA who had received the diagnosis within the past year in order to minimise recall bias. The questionnaire contained a wide range of questions regarding many potential environmental risk factors and no section in the questionnaire was given pre-eminent focus. Moreover, no previous reports have been conducted investigating the influence of night shift work on RA risk, and the prospective study on shift work and RA risk was only recently published. Therefore, the quality of the reported information on shift work and night shift work would probably not differ between cases and controls and hence tend to bias the studied association towards the null value when comparing exposed to unexposed.

Selection out of shift work has been observed in several occupational studies. ${ }^{35}$ If dropout from night shift work due to illness was a major factor, then we would expect a lower OR among current night workers than among past night workers. However, there was no significant difference between the two groups. Furthermore, the association between permanent night shift work and decreased RA risk remained unchanged when we only considered night shift work 5 years prior to the index year, indicating that the finding was not due to reverse causation.

The proportion of respondents with regard to participation in EIRA was $92 \%$ for cases and $75 \%$ for controls. A potential selection bias may result from the proportion of non-responders among the controls. However, this bias is most likely to be modest since the prevalence of shift work among the controls at different age groups was similar to what has been observed in other representative samples from Sweden. ${ }^{36}$ Moreover, the prevalence of smoking among the controls, seen as an indicator of life style, was in line with that of the general population at equivalent ages. $^{36}$

In conclusion, day-oriented shift work and rotating shift work were associated with an increased risk of ACPA-positive RA, but not ACPA-negative RA, whereas an inverse association was observed between permanent night shift work and risk of developing both subsets of RA. The mechanisms behind these observations remain to be elucidated. Sleep restriction as a consequence of shift work is associated with enhanced proinflammatory responses and may play a role in the onset of RA, whereas decreased melatonin production in night shift workers may contribute to explaining the inverse relationship between night shift work and RA risk.

Contributors AKH, LK and LA were involved in the conception and design of the study. Drafting of the manuscript and tables were done by AKH. All authors equally contributed to acquisition and analysis of the data, revised the manuscript and provided final approval of the manuscript.

Funding This work was supported by grants from the Swedish Medical Research Council, the Swedish Research Council for Health, Working Life and Welfare, King Gustaf V's 80-year foundation, Nordforsk, the Swedish Rheumatic Foundation, 
the Stockholm County Council, the Insurance Company AFA and the Innovative Medicines Initiative-supported BTCure project.

Competing interests None declared.

Patient consent Obtained.

Ethics approval The ethics committee of the Karolinska Institutet.

Provenance and peer review Not commissioned; externally peer reviewed.

Data sharing statement The datasets used and/or analysed during the current study available from the corresponding author on reasonable request.

Open Access This is an Open Access article distributed in accordance with the Creative Commons Attribution Non Commercial (CC BY-NC 4.0) license, which permits others to distribute, remix, adapt, build upon this work non-commercially, and license their derivative works on different terms, provided the original work is properly cited and the use is non-commercial. See: http://creativecommons.org/ licenses/by-nc/4.0/

(C) Article author(s) (or their employer(s) unless otherwise stated in the text of the article) 2017. All rights reserved. No commercial use is permitted unless otherwise expressly granted.

\section{REFERENCES}

1. Gibbs JE, Ray DW. The role of the circadian clock in rheumatoid arthritis. Arthritis Res Ther 2013:15:205.

2. Avouac J, Gossec L, Dougados M. Diagnostic and predictive value of anti-cyclic citrullinated protein antibodies in rheumatoid arthritis: a systematic literature review. Ann Rheum Dis 2006;65:845-51.

3. Schellekens GA, Visser H, de Jong BA, et al. The diagnostic properties of rheumatoid arthritis antibodies recognizing a cyclic citrullinated peptide. Arthritis Rheum 2000;43:155-63.

4. Kallberg H, Padyukov L, Plenge RM, et al. Gene-gene and geneenvironment interactions involving HLA-DRB1, PTPN22, and smoking in two subsets of rheumatoid arthritis. Am J Hum Genet 2007;80:867-75.

5. Padyukov L, Seielstad M, Ong RT, et al. A genome-wide association study suggests contrasting associations in ACPA-positive versus ACPA-negative rheumatoid arthritis. Ann Rheum Dis 2011;70:259-65.

6. Klareskog L, Stolt P, Lundberg K, et al. A new model for an etiology of rheumatoid arthritis: smoking may trigger HLA-DR (shared epitope)-restricted immune reactions to autoantigens modified by citrullination. Arthritis Rheum 2006;54:38-46.

7. Karlson EW, Chang SC, Cui J, et al. Gene-environment interaction between HLA-DRB1 shared epitope and heavy cigarette smoking in predicting incident rheumatoid arthritis. Ann Rheum Dis 2010;69:54-60.

8. Stolt $P$, Yahya A, Bengtsson $C$, et al. Silica exposure among male current smokers is associated with a high risk of developing ACPApositive rheumatoid arthritis. Ann Rheum Dis 2010;69:1072-6.

9. Magrini A, Pietroiusti A, Coppeta L, et al. Shift work and autoimmune thyroid disorders. Int J Immunopathol Pharmacol 2006;19:31-6.

10. Hedström AK, Åkerstedt T, Hillert J, et al. Shift work at young age is associated with increased risk for multiple sclerosis. Ann Neurol 2011;70:733-41.

11. Hedström AK, Åkerstedt T, Olsson T, et al. Shift work influences multiple sclerosis risk. Mult Scler 2015;21:1195-9.

12. Ulhôa MA, Marqueze EC, Burgos LG, et al. Shift work and endocrine disorders. Int J Endocrinol 2015;2015:1-11.

13. Bøggild $H$, Knutsson A. Shift work, risk factors and cardiovascular disease. Scand J Work Environ Health 1999;25:85-99.
14. He C, Anand ST, Ebell MH, et al. Circadian disrupting exposures and breast cancer risk: a meta-analysis. Int Arch Occup Environ Health 2015;88:533-47.

15. Puttonen S, Oksanen T, Vahtera J, et al. Is shift work a risk factor for rheumatoid arthritis? The finnish public sector study. Ann Rheum Dis 2010;69:679-80.

16. Yoshida K, Hashimoto T, Sakai Y, et al. Involvement of the circadian rhythm and inflammatory cytokines in the pathogenesis of rheumatoid arthritis. J Immunol Res 2014;2014:1-6.

17. Spies CM, Hoff P, Mazuch J, et al. Circadian rhythms of cellular immunity in rheumatoid arthritis: a hypothesis-generating study. Clin Exp Rheumatol 2015;33:34-43.

18. Davis S, Mirick DK, Chen C, et al. Night shift work and hormone levels in women. Cancer Epidemiol Biomarkers Prev 2012;21:609-18.

19. Marie Hansen A, Helene Garde A, Hansen J. Diurnal urinary 6-sulfatoxymelatonin levels among healthy Danish nurses during work and leisure time. Chronobiol Int 2006;23:1203-15.

20. Mirick DK, Bhatti P, Chen C, et al. Night shift work and levels of 6-sulfatoxymelatonin and cortisol in men. Cancer Epidemiol Biomarkers Prev 2013:22:1079-87.

21. Yamauchi $\mathrm{H}$. Effects of night work on urinary excretion rates of 6-sulfatoxymelatonin, norepinephrine and estriol in pregnant women. Ind Health 2004;42:268-76.

22. Dumont M, Paquet J. Progressive decrease of melatonin production over consecutive days of simulated night work. Chronobiol Int 2014;31:1231-8.

23. Dubucquoi S, Solau-Gervais E, Lefranc D, et al. Evaluation of anticitrullinated filaggrin antibodies as hallmarks for the diagnosis of rheumatic diseases. Ann Rheum Dis 2004;63:415-9.

24. Akerstedt T. Shift work and disturbed sleep/wakefulness. Occup Med 2003;53:89-94.

25. Bollinger T, Bollinger A, Skrum L, et al. Sleep-dependent activity of $T$ cells and regulatory T cells. Clin Exp Immunol 2009;155:231-8.

26. Bollinger $\mathrm{T}$, Bollinger $\mathrm{A}$, Oster $\mathrm{H}$, et al. Sleep, immunity, and circadian clocks: a mechanistic model. Gerontology 2010;56:574-80.

27. Zielinski MR, Krueger JM. Sleep and innate immunity. Front Biosci;3022:632-42.

28. van Leeuwen WM, Lehto $M$, Karisola $\mathrm{P}$, et al. Sleep restriction increases the risk of developing cardiovascular diseases by augmenting proinflammatory responses through IL-17 and CRP. PLoS One 2009;4:e4589.

29. Kimura A, Kishimoto T. IL-6: regulator of Treg/Th17 balance. Eur J Immunol 2010;40:1830-5.

30. Maestroni GJ, Sulli A, Pizzorni C, et al. Melatonin in rheumatoid arthritis: synovial macrophages show melatonin receptors. Ann NY Acad Sci 2002:966:271-5.

31. Sulli A, Maestroni GJ, Villaggio B, et al. Melatonin serum levels in rheumatoid arthritis. Ann N Y Acad Sci 2002;966:276-83.

32. Cutolo M, Maestroni GJ, Otsa K, et al. Circadian melatonin and cortisol levels in rheumatoid arthritis patients in winter time: a north and south Europe comparison. Ann Rheum Dis 2005;64:212-6.

33. Hansson I, Holmdahl R, Mattsson R. Constant darkness enhances autoimmunity to type II collagen and exaggerates development of collagen-induced arthritis in DBA/1 mice. J Neuroimmunol 1990;27:79-84.

34. Hansson I, Holmdahl R, Mattsson R. The pineal hormone melatonin exaggerates development of collagen-induced arthritis in mice. $J$ Neuroimmunol 1992;39:23-30.

35. Checkoway H, Pearce NE, Crawford-Brown DJ. Research methods in occupational epidemiology. New York: Oxford University Press, 1989

36. Internet-based information. http://www.scb.se (accessed $26 \mathrm{Jul}$ 2015). 